\title{
Absolute configuration directly determined from 3D electron diffraction data
}

\author{
P.B. Klar ${ }^{1}$, P. Brázda ${ }^{1}$, Y. Krysiak ${ }^{1,2}$, M. Klementová1 ${ }^{1}$ L. Palatinus ${ }^{1}$ \\ ${ }^{1}$ Institute of Physics of the Czech Academy of Sciences; Prague, Czech Republic \\ ${ }^{2}$ Institute of Inorganic Chemistry of the Leibniz University Hannover; Hannover, Germany
}

klar@fzu.cz

Multiple scattering in 3D electron diffraction (3D ED) experiments is responsible for deviations of diffracted intensities from intensities expected from kinematical diffraction theory [1]. Though this is usually considered a disturbing factor in routine structure determinations, these deviations also contain valuable information on the absolute structure [2]. Analysing 3D ED measurements from different laboratories around the world, we demonstrate that the absolute structure of single submicrometric crystals can be reliably and easily determined in a routine way if dynamical diffraction effects are incorporated in the refinement of the structure model.

We investigated and reinvestigated data sets of non-centrosymmetric samples recorded with beam-precession (precession-assisted 3D ED) and with continuous-rotation 3D ED (IEDT, MicroED, cRED) to determine the absolute structure, which directly determines the absolute configuration of chiral molecules in the unit cell. Dynamical effects are very sensitive to the absolute structure due to the interference of multiple beams contributing to each reflection [3]. In comparison to X-ray diffraction-based methods, the requirements for a successful determination of the absolute structure are strongly reduced. We demonstrate that with a completeness as low as $25 \%$ (Figure 1), a limited resolution $d_{\min }>1 \AA$ and only a preliminary structure model the correct chirality can still be identified. The low requirements also allow significantly reducing the number of refinement parameters so that the computationally demanding calculations applying dynamical diffraction theory are only a matter of minutes even for unit cells with a volume of several thousand $\AA^{3}$. The determination is based on a simple comparison of residual factors $\left(R_{\mathrm{obs}}\right.$ and $\left.w R_{\mathrm{all}}\right)$ of the refined, inversion-related models (Figure 1). With this approach, the routine determination of the chirality of molecules in submicrometric crystals is ready to be implemented in any laboratory with access to 3D electron diffraction measurements. Considering ongoing developments, improvements and increasing level of automatization of data acquisition and analysis [1], we believe that especially the pharmaceutical industry will strongly benefit from the presented approach.

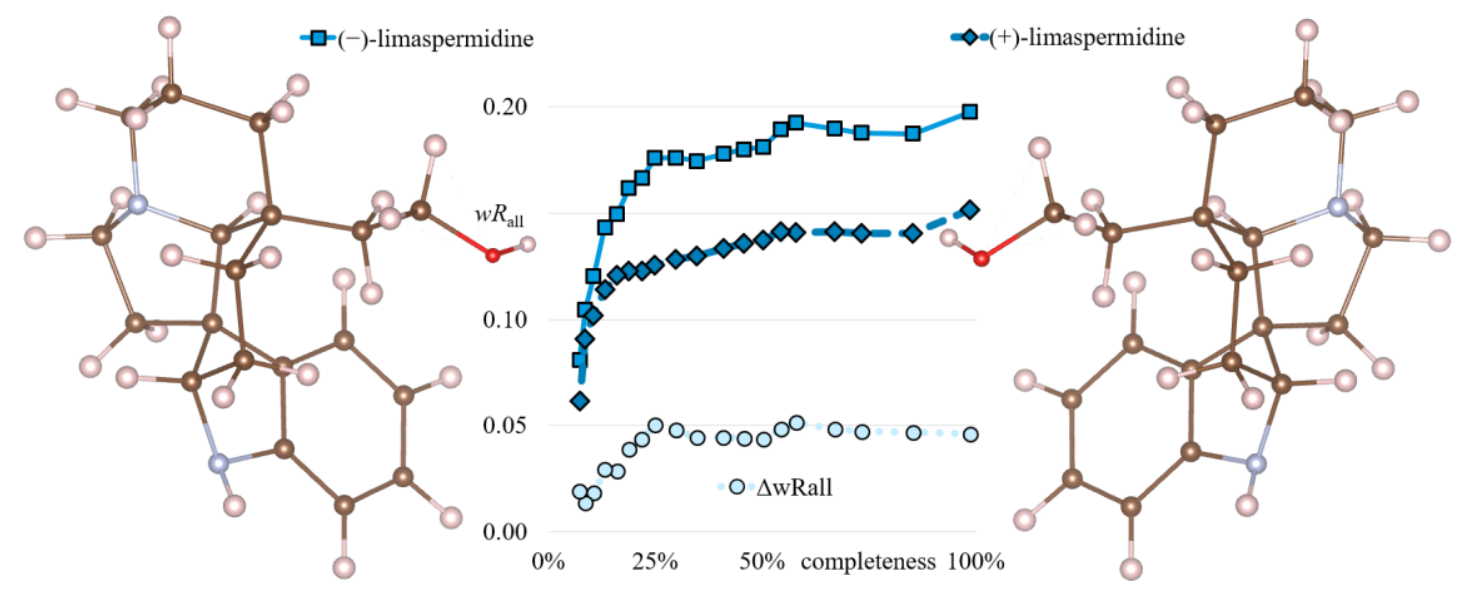

Figure 1. Identification of (+)-limaspermidine by comparing $w R_{\text {all }}$ of refinements with different completeness. Even with low completeness data sets, the identification is straightforward as refinements of (-)-limaspermidine have a clearly higher $w R_{\text {all }}$.

[1] Gemmi, M., Mugnaioli, E., Gorelik, T., Kolb, U., et al. (2019). ACS Cent. Sci. 5, 1315-1329.

[2] Brázda, P., Palatinus, L., Babor, M. (2019). Science. 364, 667-669

[3] Spence, J.C.H., Zuo, J.M., O'Keeffe, M., Marthinsen, K., Hoier, R. (1994). Acta. Cryst. A50, 647-650

Keywords: absolute structure; absolute configuration; chirality; 3D electron diffraction; MicroED

Support by the Czech Science Foundation (project number 21-05926X), and by Operational Programme Research, Development and Education financed by European Structural and Investment Funds and the Czech Ministry of Education, Youth and Sports (Project No. SOLID21 CZ.02.1.01/0.0/0.0/16_019/0000760) is highly appreciated.

Acta Cryst. (2021), A77, C210 